\title{
Diagnosis and rehabilitation of a pregnant woman with spinal cord disorder due to spinal cord tumor
}

\author{
Jason Bitterman ${ }^{1} \cdot$ Jayne Donovan ${ }^{1,2} \cdot$ Mylan Lam $^{2}$
}

Received: 11 December 2018 / Revised: 10 January 2019 / Accepted: 12 January 2019

(c) International Spinal Cord Society 2019

\begin{abstract}
Introduction Spinal cord tumors are a rare cause of spinal cord disorder (SCD). Early diagnosis can be challenging, especially in patient populations with other potential etiologies for back pain, such as pregnant women.

Case presentation A 28-year-old female presented at 8 weeks gestation with thoracic back pain initially diagnosed as gestational low back pain. Her symptoms progressed to lower extremity weakness. After diagnosis and resection of a spinal cord ependymoma, we collaborated with the inpatient interdisciplinary team to devise a rehabilitation program focused on minimizing pregnancy-related complications and optimizing care of a newborn in the setting of a new paraplegia.

Discussion Providers should not assume all pregnant women with low back pain have gestational back pain, as it can be due to more insidious causes. The rehabilitation course for women who develop an SCD during gestation should be carefully tailored toward their pre- and post-natal care and with the education of how the disorder can affect pregnancy and parenting.
\end{abstract}

\section{Introduction}

Back pain is a common complaint during pregnancy, usually during the second and third trimesters in a lumbar or sacroiliac pattern [1]. However, physicians should keep a broad differential when a pregnant patient presents with low back pain. Ependymomas are the most common intramedullary spinal cord neoplasms in adults [2] but can be difficult to diagnosis early due to their slow-growing nature with an initial presentation of generalized back pain. Nevertheless, there are certain insidious signs and symptoms that should raise suspicions for spinal neoplasms.

This case report illustrates the challenges of diagnosing and rehabilitating a pregnant woman with a spinal cord disorder (SCD) due to an ependymoma. Caring for pregnant individuals with SCD can be particularly difficult as those with experience with SCD may have limited experience with obstetrics, and vice versa. Women with SCD report poor knowledge of how their disorder affects

Jason Bitterman

jsb296@njms.rutgers.edu

1 Department of Physical Medicine \& Rehabilitation, Rutgers New Jersey Medical School, Newark, NJ, USA

2 Kessler Institute for Rehabilitation, West Orange, NJ, USA pregnancy [3, 4]. Providers should be well versed in how SCD can influence pre-natal, peri-natal, and post-natal care to best prepare their patients and prevent obstetric complications.

\section{Case presentation}

A 28-year-old female with a history of a motor vehicle accident 4 years earlier with chronic lumbar back pain developed new thoracic level back pain at 8 weeks gestation. The pain was localized to the left of the thoracic spine and radiated to her anterior left ribs. The pain was exacerbated by lying supine. For her new symptoms, the patient was initially seen by her primary care physician and a chiropractor. Both attributed her back pain to residual musculoskeletal strain from her prior motor vehicle accident and pregnancy-related back pain. She was referred for physical therapy; however, she was unable to tolerate therapy beyond one session due to progressively worsening pain.

At 14 weeks gestation, the patient began to develop weakness in her right foot. Over the next 2 weeks, she began having paresthesias of the right lower extremity and her weakness progressed to her right hip. Her ambulation deteriorated and she required a cane and eventually a rolling walker to ambulate. She saw a neurologist at 18 weeks 
gestation who noted decreased sensation on the plantar right foot and decreased strength in the right lower extremity. A magnetic resonance imaging revealed an expansile, partially cystic intramedullary thoracic cord mass at T7-T9. The patient then underwent emergent T5-T8 laminectomy with mass resection; the pathology report later confirmed a diagnosis of spinal ependymoma.

Following surgery, at 20 weeks gestation, the patient was admitted for acute inpatient rehabilitation. Her admission exam demonstrated $1 / 5$ strength in the right hip flexors and $2 / 5$ strength in the distal lower extremity key muscle groups (as defined according to the International Standards for Neurological Classification of Spinal Cord Injury (ISNCSCI)) [5] and 2/5 strength in the key muscle groups of the left lower extremity. She had impaired sensation to light touch and pinprick in the bilateral lower extremities from the L3 dermatome distally with evidence of sacral sparing and diagnosed with L1 AIS C paraplegia.

An individualized rehabilitation program was tailored toward achieving functional independence, minimizing pregnancy-related complications, and planning for the care of a newborn in the context of her new SCD. Occupational therapy trained her in newborn care while having limited lower extremity strength, with strategies for safe feeding, diaper changing, carrying of the child, and transferring of the child. Physical therapy trained the patient in managing a newborn in a wheelchair and, as the patient's strength improved, with a rolling walker.

While admitted for inpatient rehabilitation, the patient was educated on how an SCD can affect pregnancy. Initial goals were set for independence at a manual wheelchair level. As her strength increased, this was adjusted to ambulation with an assistive device. Psychology assisted with adjustment to her injury and spinal tumor diagnosis. The patient's obstetrician was updated on her progress.

After 3 weeks of inpatient rehabilitation, the patient's strength had improved with 4/5 left knee extensors, 3/5 right knee extensors, and 3/5 left ankle dorsiflexors and plantarflexors Throughout her course, she had normal bowel and bladder function with awareness of need to empty the bladder and move her bowels, continence of urine and stool, ability to empty the bladder without requiring catheterization, and voluntary sphincter contraction [6]. By the day of discharge, the patient was ambulating independently with a rolling walker.

\section{Discussion}

Back pain is a common complaint during pregnancy, with the prevalence of gestational low back pain ranging from 50 to $80 \%$ [7]. Gestational back pain and pelvic girdle pain are due to increased joint laxity and biomechanical changes. Persistently elevated levels of progesterone and estrogen from the late first trimester onward cause increased the mobility of joints [1]. The enlarging uterus also weakens the abdominal musculature, causing strain on the lumbar muscles to maintain core stability. The pelvis anteriorly rotates due to the gravid uterus and hypermobile joints, leading to a compensatory lumbar hyperlordosis, adding further flexion force on the lumbar spine. This puts more strain on the low back and pelvis [1].

This patient's symptoms suggested a more insidious etiology, even before her neurologic changes. Her pain began at 8 weeks gestation; an earlier presentation for pregnancy-related back pain, which usually presents between the 18th and 36th weeks of gestation. Furthermore, her pain was specifically at the thoracic level, while pregnancy-related pain is typically localized to the lumbar spine, sacroiliac joints, and pelvic girdle. The early presentation and thoracic location of this patient's symptoms did not fit with the biomechanical history seen with pregnancy-related back pain. In addition to this, back pain worse while recumbent also raises concerns for a spinal lesion. Spinal tumors have been reported to initially be misdiagnosed until the occurrence of neurologic abnormalities [8].

The incidence of all cancers in pregnancy is uncommon, occurring in about 1 in 1000 pregnancies [9]. For spine tumors in pregnancy, a recent report found that giant cell tumors, hemangiomas, and schwannomas were the most frequent tumor types [10]. There have been case reports describing these tumors along with spinal meningiomas and ependymomas $[8,11-15]$. Spinal ependymomas are rare benign, slow-growing tumors that can present with back pain and neurologic changes depending on their location within the cord $[2,15,16]$. Patients may have residual functional deficits after surgical resection, although it is difficult to predict. One study assessing functional outcomes after ependymoma resection found that $34 \%$ of patients who were non-ambulant pre-operatively remained non-ambulant post-operatively. Shorter duration of symptoms prior to surgery was the only predictor for post-operative ambulation [16]. Another study assessing outcomes found that the only predictor for the outcome was tumor location, with the lumbar region having the best early outcomes [2].

In the rehabilitation setting, women should be properly educated on how their SCD can affect pregnancy, delivery, and care of their child. Furthermore, collaboration of the SCD team with the patient's obstetrics team is key in ensuring success, as the latter may not be as experienced with the SCD patient population. Below are some key aspects of antenatal, delivery, and postnatal care to discuss with patients with SCD. 
- Bladder: The enlarging uterus can lead to more incontinence, requiring either more frequent intermittent catheterizations or placement of an indwelling catheter [17]. Women are more likely to have urinary tract infections and pyelonephritis as both neurogenic bladder from SCD and pregnancy itself increase the risk [18, 19].

- Bowel: Patients may need to adjust their bowel regimen as pregnancy increases their risk of constipation [20].

- Skin: Immobilization, weight gain, and edema secondary to pregnancy can increase the risk of pressure injuries $[18,19]$. A cohort study of this population found $8.8 \%$ of women developed decubitus pressure injuries during pregnancy [17].

- Sensation of fetal movements and uterine contractions: Women with lesions above T11 will have abnormal or absent sensation of the uterus. They should be taught how to palpate their abdomen to detect these movements and contractions. These patients are also at increased risk for breech or transverse positioning of the fetus, possibly due to reduced abdominal muscle tone [18].

- Autonomic dysreflexia (AD): In patients with lesions at T6 and above, AD can lead to uteroplacental vasoconstriction, fetal hypoxemia, and fetal bradycardia. AD can occur with vaginal exams, uterine contractions, and during labor [18]. A cohort study found $60 \%$ of women with SCI above T6 had an episode of AD during pregnancy [17]. Whenever $\mathrm{AD}$ is being considered in a pregnant woman, physicians must rule out preeclampsia as it also presents with rising blood pressure [19].

- Analgesia during labor: Patients at risk for $\mathrm{AD}$ are recommended to receive epidural analgesia once labor is diagnosed [18]. Otherwise, the decision is up to the patient. Those with lesions between T6 and T10 may not require any analgesia as uterine sensory innervation derives from T11-L1 roots.

- Delivery: Vaginal delivery is possible in SCI. Some recommend performing $\mathrm{C}$-sections in women with syringomyelia as straining may worsen the cyst due to increased intraspinal pressure [21]. Otherwise, Csections should only be performed for obstetric indications.

- Neonatal complications: There is no difference in the incidence of congenital malformations, preterm labor, and neonatal mortality between patients with and without SCD [17, 18].

- Breastfeeding: Patient with lesions above T7 may have delayed initiation of breastfeeding and may require additional stimulation [22]. Otherwise, women should be able to breastfeed without complications.

This patient showed improvement in function over the duration of her rehabilitation course. A recent analysis of the U.S. National Spinal Cord Injury Database examined the relationship of pregnancy and functional outcomes [23]. It found that women classified as "current pregnancy" (defined as hospitalized in the 12 months prior to data collection for childbirth or complications of pregnancy) had higher discharge Functional Independence Measure (FIM) motor scores, physical independence, and mobility compared to nonpregnant women with a spinal cord injury.

This case demonstrates the care needed to properly diagnose a spinal tumor in populations expected to have back pain. Furthermore, it illustrates the utility of tailoring rehab to a patient's specific needs (in this case, pre-, peri-, and post-natal care) and providers role as educators. Women with SCD likely want to know how their disorder affects fertility and pregnancy. Providers must be knowledgeable and proactive in teaching women to assuage potential worries and support them.

\section{Compliance with ethical standards}

Conflict of interest The authors declare that they have no conflict of interest.

Publisher's note: Springer Nature remains neutral with regard to jurisdictional claims in published maps and institutional affiliations.

\section{References}

1. Casagrande D, Gugala Z, Clark SM, Lindsey RW. Low back pain and pelvic girdle pain in pregnancy. J Am Acad Orthop Surg. 2015;23:539-49.

2. Domazet I, Pasalic I, Nemir J, Peterkovic V, Vukic M. Predictors of functional outcome after spinal ependymoma resection. J Neurosci Rural Pract. 2018;9:354-8.

3. Ghidini A, Healey A, Andreani M, Simonson MR. Pregnancy and women with spinal cord injuries. Acta Obstet Gynecol Scand. 2008;87:1006-10.

4. Arsh A, Darain H, Ilyas SM, Zeb A. Consequences of traumatic spinal cord injury during pregnancy in Pakistan. Spinal Cord Ser Cases. 2017;3:17041.

5. International Standards for Neurological Classification of Spinal Cord Injury (ISNCSCI). 2015. Available from: https://asia-spina linjury.org/learning/.

6. Krassioukov A, Biering-Sørensen F, Donovan W, Kennelly M, Kirshblum S, Krogh K, et al. International standards to document remaining autonomic function after spinal cord injury. J Spinal Cord Med. 2012;35:202-11. https://doi.org/10.1179/ 1079026812Z.00000000053.

7. Sabino J, Grauer JN. Pregnancy and low back pain. Curr Rev Musculoskelet Med. 2008;1:137-41.

8. Fujii K, Orisaka M, Yamamoto M, Nishijima K, Yoshida Y. Primary intramedullary spinal cord tumour in pregnancy: a case report. Spinal Cord Ser Cases. 2018;4:25.

9. Pavlidis NA. Coexistence of pregnancy and malignancy. Oncologist. 2002;7:279-87.

10. Meng T, Yin H, Li Z, Li B, Zhou W, Wang J, et al. Therapeutic strategy and outcome of spine tumors in pregnancy: a report of 21 cases and literature review. Spine. 2015;40:E146-53. 
11. Han IH, Kuh SU, Kim JH, Chin DK, Kim KS, Yoon YS, et al. Clinical approach and surgical strategy for spinal diseases in pregnant women: a report of ten cases. Spine. 2008;33:E614-9.

12. Kathiresan AS, Johnson JN, Hood BJ, Montoya SP, Vanni S, Gonzalez-Quintero VH. Giant cell bone tumor of the thoracic spine presenting in late pregnancy. Obstet Gynecol. 2011;118:428-31.

13. Pikis S, Cohen JE, Rosenthal G, Barzilay Y, Kaplan L, Shoshan $\mathrm{Y}$, et al. Spinal meningioma becoming symptomatic in the third trimester of pregnancy. J Clin Neurosci. 2013;20:1797-9.

14. Slimani O, Jayi S, Fdili Alaoui F, Bouguern H, Chaara H, Fikri G, et al. An aggressive vertebral hemangioma in pregnancy: a case report. J Med Case Rep. 2014;8:207.

15. van der Hoeven MEM, de Pont LMH, Koppen H. Severe nightly thoracic pain presenting during pregnancy: a case report. Case Rep Neurol. 2018;10:135-9.

16. Gavin Quigley D, Farooqi N, Pigott TJ, Findlay GF, Pillay R, Buxton N, et al. Outcome predictors in the management of spinal cord ependymoma. Eur Spine J. 2007;16:399-404.

17. Le Liepvre H, Dinh A, Idiard-Chamois B, Chartier-Kastler E, Phe $\mathrm{V}$, Even A, et al. Pregnancy in spinal cord-injured women, a cohort study of 37 pregnancies in 25 women. Spinal Cord. 2017;55:167-71.

18. Dawood R, Altanis E, Ribes-Pastor P, Ashworth F. Pregnancy and spinal cord injury. Obstet Gynaecol. 2014;16:99-107.

19. Castro JS, Lourenco C, Carrilho M. Successful pregnancy in a woman with paraplegia. BMJ Case Rep. 2014;2014: bcr2013202479.

20. Camune BD. Challenges in the management of the pregnant woman with spinal cord injury. J Perinat Neonatal Nurs. 2013;27:225-31.

21. Daskalakis GJ, Katsetos CN, Papageorgiou IS, Antsaklis AJ, Vogas EK, Grivachevski VI, et al. Syringomyelia and pregnancy - case report. Eur J Obstet Gynecol Reprod Biol. 2001;97:98-100.

22. Cowley KC. Psychogenic and pharmacologic induction of the letdown reflex can facilitate breastfeeding by tetraplegic women: a report of 3 cases. Arch Phys Med Rehabil. 2005;86:1261-4.

23. Iezzoni LI, Chen Y, McLain AB. Current pregnancy among women with spinal cord injury: findings from the US national spinal cord injury database. Spinal Cord. 2015;53:821-6. 